\title{
RELATIVELY STABLE BUNDLES OVER ELLIPTIC FIBRATIONS
}

\author{
Claudio Bartocci $\ddagger$ \\ Ugo Bruzzo $\S \star$ \\ Daniel Hernández Ruipérez , José M. Muñoz Porras $₫$ \\ †Dipartimento di Matematica, Università di Genova, \\ Via Dodecaneso 35, 16146 Genova, Italy \\ $\S$ Scuola Internazionale Superiore di Studi Avanzati, \\ Via Beirut 2-4, 34014 Trieste, Italy \\ $\star$ Istituto Nazionale di Fisica Nucleare, \\ Sezione di Trieste \\ ฯ Departamento de Matemáticas, Universidad de Salamanca, \\ Plaza de la Merced 1-4, 37008 Salamanca, Spain \\ bartocci@dima.unige.it, bruzzo@sissa.it, \\ ruiperez@usal.es, jmp@usal.es
}

20 February 2000, Revised 25 August 2001 and 13 September 2004

\begin{abstract}
We consider a relative Fourier-Mukai transform defined on elliptic fibrations over an arbitrary base scheme. This is used to construct relative Atiyah sheaves and generalize Atiyah's and Tu's results about semistable sheaves over elliptic curves to the case of elliptic fibrations. Moreover we show that this transform preserves relative (semi)stability of sheaves of positive relative degree.
\end{abstract}

This research was partially supported by the Spanish DGES through the research project PB961305, by "Junta de Castilla y León" through the research project SA27/98, by GNSAGA (Italian National Research Council) and by an Italian-Spanish cooperation project. The authors are members of VBAC (Vector Bundles on Algebraic Curves), which is partially supported by EAGER (EC FP5 Contract no. HPRN-CT-2000-00099) and Edge (EC FP5 Contract no. HPRN-CT2000-00101). 


\section{INTRODUCTION}

In 44 we defined a relative Fourier-Mukai transform on elliptic K3 surfaces. A relative Fourier-Mukai transform for abelian schemes had been considered by Mukai [13 generalizing his previous definition holding for abelian varieties [12. The case of (absolute) transforms for K3 surfaces can be found in [3] and [14].

In [10] a relative Fourier-Mukai transform was introduced on elliptic fibrations $p: X \rightarrow B$ over an arbitrary normal base scheme $B$ (see also [5, 6] for the case of elliptic surfaces and that of elliptic fibrations over smooth bases). In this paper we prove that this transform preserves the relative stability of the sheaves on $X$ of positive relative degree. This, together with the results in [10], generalizes to elliptic fibrations the classical theory of moduli spaces of vector bundles on elliptic curves [1, 16].

As it is well known, every indecomposable vector bundle over a smooth elliptic curve $C$ is semistable, and stability holds if and only if rank and degree are relatively prime. This result is proved in [16] by showing that the Atiyah bundle $\mathcal{A}_{n}$ on $C$ is semistable. The Atiyah bundle $\mathcal{A}_{n}$ is the rank $n$ indecomposable vector bundle defined inductively by the exact sequence

$$
0 \rightarrow \mathcal{O}_{C} \rightarrow \mathcal{A}_{n} \rightarrow \mathcal{A}_{n-1} \rightarrow 0
$$

where $\mathcal{A}_{0}=\mathcal{O}_{C}$. These constructions carry over to the case of elliptic curves with a singular point. As a direct consequence of this characterization, the moduli space of semistable bundles of rank $n$ and degree $d$ is naturally isomorphic to the symmetric product $S^{h} C$, where $h$ is the g.c.d. of $n$ and $d$.

It turns out that these results can be extended to the case of elliptic fibrations as it is shown in [10] and in the present paper (see also [5, 6]). In our construction the relative Fourier-Mukai transform plays a central role. In particular, this transform allows us to give a natural definition of the relative rank $i$ Atiyah sheaf $\mathcal{A}_{i}$ (Prop. 2.9] and Def. [2.11) and to obtain explicitly the isomorphism between the compactified relative Jacobian $\bar{J}_{n}$ of (flat families of) torsion-free, rank 1 and degree $n$ sheaves and the moduli space $\mathcal{M}(n,-1)$ of rank $n$ relatively stable sheaves of degree -1 (Cor. 3.4).

Moreover, the results in this paper provide a kind of duality between the following functors.

(1) The first functor will be denoted by $\mathbf{F}_{X}^{i}(n, d)$; it associates to every scheme $S$ over $B$ the scheme parametrizing exact sequences of sheaves on $X_{S}$

$$
0 \rightarrow \mathrm{E} \otimes \mathcal{A}_{i} \rightarrow \mathcal{E}_{n} \rightarrow \mathcal{E}_{n-i} \rightarrow 0
$$

where $\mathrm{E}$ is torsion-free, of rank 1 and relative degree zero and $\mathcal{E}_{n}, \mathcal{E}_{n-i}$ are relatively stable sheaves of relative degree $d \geq 0$ and rank $n$ and $n-i$, respectively.

(2) The second functor, denoted by $\mathbf{H}_{X}^{i}(r, d)$, associates to $S$ the scheme parametrizing the exact sequences of sheaves on $X_{S}$

$$
0 \rightarrow \mathcal{E}_{r} \rightarrow \mathcal{E}_{r}^{\prime} \rightarrow \mathcal{K}(x) \rightarrow 0,
$$


where $\mathcal{E}_{r}, \mathcal{E}_{r}^{\prime}$ are relatively stable sheaves of rank $r$ and $\mathcal{K}(x)$ has length $i$ on every fibre and is concentrated on the image $x(S)$ of a section $x: S \hookrightarrow X_{S}$ of $p_{S} ; d$ denotes the relative degree of $\mathcal{E}_{r}$.

In Theorem 4.3 we prove that the Fourier-Mukai transform establishes an isomorphism between the functors $\mathbf{F}_{X}^{i}(n, d)$ and $\mathbf{H}_{\widehat{X}}^{i}(d,-n)$, where $\widehat{X}$ is the compactified relative Jacobian of $X$.

Acknowledgement. We thank Igor Burban and Bernd Kreussler for pointing out that in a previous version of this paper Proposition 2.9 was incorrectly stated.

\section{Preliminaries}

Let $p: X \rightarrow B$ be an elliptic fibration, where $B$ is a normal algebraic scheme over an algebraically closed field, and the fibres of the projection $p$ are geometrically integral Gorenstein curves of arithmetic genus 1. We also assume that $p$ has a section $e$ taking values in the smooth locus of $p$. We write $H=e(B)$; this is a relative polarization. We shall also denote by $X_{t}$ the fibre of $p$ over $t \in B$.

The sheaf $\omega=R^{1} p_{*} \varnothing_{X}$ is a line bundle on $B$. By relative duality, $\omega \simeq\left(p_{*} \omega_{X / B}\right)^{*}$, where $\omega_{X / B}$ is the relative dualizing sheaf, and also $\omega_{X / B} \simeq p^{*} \omega^{-1}$.

We denote by $\widehat{X}$ the compactified relative Jacobian of $X$. This is the scheme representing the functor which to any scheme morphism $f: S \rightarrow B$ associates the space of equivalence classes of $S$-flat sheaves on $p_{S}: X \times_{B} S \rightarrow S$, whose restrictions to the fibres of $f$ are torsion-free, of rank one and degree zero; two such sheaves $\mathcal{F}, \mathcal{F}^{\prime}$ are considered to be equivalent if $\mathcal{F}^{\prime} \simeq \mathcal{F} \otimes p_{S}^{*} \mathcal{N}$ for a line bundle $\mathcal{N}$ on $S$ (cf. [2]).

There is a natural isomorphism of $B$-schemes

$$
\begin{aligned}
\varpi: X & \rightarrow \widehat{X} \\
x & \mapsto \mathfrak{m}_{x}^{*} \otimes \varnothing_{X_{t}}(-e(t))
\end{aligned}
$$

where $\mathfrak{m}_{x}$ is the ideal sheaf of the point $x$ in $X_{t}$. The section $e$ induces then a section $\hat{e}$ of $\hat{p}: \widehat{X} \rightarrow B$ whose image is a relative polarization $\Theta$.

We can normalize the Poincaré sheaf $\mathcal{P}$ on $X \times_{B} \widehat{X}$ so that

$$
\mathcal{P}_{\mid H \times_{B} \widehat{X}} \simeq \emptyset_{\widehat{X}}
$$

One has

$$
\mathcal{P} \simeq \mathcal{J} \otimes \pi^{*} \emptyset_{X}(H) \otimes \hat{\pi}^{*} \emptyset_{\widehat{X}}(\Theta) \otimes q^{*} \omega^{-1},
$$

where $\mathcal{J}$ is the ideal of the graph $\gamma: X \hookrightarrow X \times_{B} \widehat{X}$ of $\varpi: X \rightarrow \widehat{X}$ and $q=p \circ \pi=\hat{p} \circ \hat{\pi}$. Then $\mathcal{P}$ is flat over both $X$ an $\widehat{X}$. Moreover, one has:

Lemma 2.1. The dual $\mathcal{P}^{*}$ of the Poincaré sheaf is flat over both factors and $\mathcal{P}$ is reflexive, that is, $\mathcal{P} \simeq \mathcal{P}^{* *}$.

Proof. One has $\mathcal{E} x t_{\emptyset_{X_{t}}}^{1}\left(\mathcal{P}_{\xi}, \varnothing_{X_{t}}\right)=0$ for every point $\xi \in \widehat{X}$ (where $t=\tilde{p}(\xi)$ ), as it was proved in Lemma 0.4 of [8]. Now, by Theorem 1.10 of [2], $\mathcal{E} x t_{\emptyset_{X \times_{B} \hat{X}}}^{1}\left(\mathcal{P}, \varnothing_{X \times_{B} \hat{X}}\right)=0$, and the base change property for the local Ext's ([2], Theorem 1.9) implies that $\left(\mathcal{P}^{*}\right)_{\xi} \simeq\left(\mathcal{P}_{\xi}\right)^{*}$ 
for every point $\xi \in \widehat{X}$. By applying once more Theorem 1.9 of [2], we have that $\mathcal{P}^{*}$ is flat over $\widehat{X}$. After interchanging the roles of $X$ and $\widehat{X}$, we obtain that $\mathcal{P}^{*}$ is flat over $X$ as well. Then, $\mathcal{P}^{*}$ defines a morphism $\iota: \widehat{X} \rightarrow \widehat{X}$ that maps any rank-one torsion-free zero-degree coherent sheaf $\mathcal{F}$ on a fibre $X_{t}$ to its dual $\mathcal{F}^{*}$. Thus, $(1 \times \iota)^{*} \mathcal{P} \simeq \mathcal{P}^{*} \otimes \hat{\pi}^{*} \mathcal{N}$ for some line bundle $\mathcal{N}$ on $\widehat{X}$, which turns out to be trivial. Then

$$
(1 \times \iota)^{*} \mathcal{P} \simeq \mathcal{P}^{*}
$$

The morphism $\iota \circ \iota: \widehat{X} \rightarrow \widehat{X}$ is the identity on the relative Jacobian $J(X / B) \subset \widehat{X}$; by separateness $\iota \circ \iota=\mathrm{Id}$, and the isomorphism (3) implies $\mathcal{P} \simeq \mathcal{P}^{* *}$.

As a consequence, every coherent sheaf $\mathcal{F}$ on $X \times_{B} S$ flat over $S$ whose restrictions to the fibres of $X \times_{B} S \rightarrow S$ are torsion-free and of rank one and degree zero is reflexive, $\mathcal{F} \simeq \mathcal{F}^{* *}$.

For any morphism $S \rightarrow B$ of algebraic varieties we define the (relative) Fourier-Mukai transform as the functor $\mathbf{S}_{S}: D^{-}\left(X_{S}\right) \rightarrow D^{-}\left(\widehat{X}_{S}\right)$ (where $D^{-}$denotes the derived category of coherent sheaves consisting of complexes bounded from above, and a subscript $S$ denotes base change to $S$, so that $X_{S}=X \times_{B} S$ etc.) given by

$$
\mathbf{S}_{S}(F)=\mathbf{R} \hat{\pi}_{S *}\left(\pi_{S}^{*} F \stackrel{L}{\otimes} \mathcal{P}_{S}\right),
$$

$\pi: X \times_{B} \widehat{X} \rightarrow X$ and $\hat{\pi}: X \times_{B} \widehat{X} \rightarrow \widehat{X}$ being the natural projections. We can also define the Fourier-Mukai transforms of a single sheaf $\mathcal{F}$ as

$$
\mathbf{S}_{S}^{i}(\mathcal{F})=R^{i} \hat{\pi}_{S *}\left(\pi_{S}^{*} \mathcal{F} \otimes \mathcal{P}_{S}\right)
$$

One then says that a sheaf $\mathcal{F}$ on $X_{S}$ is $\mathrm{WIT}_{i}$ if

$$
\mathbf{S}_{S}^{j}(\mathcal{F})=0 \quad \text { for } j \neq i \text {. }
$$

In this case we write $\widehat{\mathcal{F}}=\mathbf{S}_{S}^{i}(\mathcal{F})$.

Several results that were proved in [4] in the case of elliptic K3 surfaces also hold under the conditions of the present paper. For the reader's convenience we recall here some of them; further details can be found in [4].

Proposition 2.2. Let $\mathcal{F}$ be a sheaf on $X_{S}$, flat over $S$. The Fourier-Mukai transforms $\mathbf{S}_{S}^{i}(\mathcal{F})$ are $S$-flat as well. Moreover, for every morphism $g: T \rightarrow S$ one has $g_{\widehat{X}}^{*} \mathbf{S}_{S}^{1}(\mathcal{F}) \simeq$ $\mathbf{S}_{T}^{1}\left(g_{X}^{*} \mathcal{F}\right)$, where $g_{X}: X_{T} \rightarrow X_{S}, g_{\widehat{X}}: \widehat{X}_{T} \rightarrow \widehat{X}_{S}$ are the morphisms induced by $g$.

Let $f: S \rightarrow B$ be a morphism and $\mathrm{E}$ a coherent sheaf on $X \times_{B} S$ flat over $S$ whose restrictions to the fibres of $p_{S}$ are torsion-free and have rank one and degree zero. Let $\phi: S \rightarrow \widehat{X}$ be the morphism determined by the universal property, so that

$$
(1 \times \phi)^{*} \mathcal{P} \simeq \mathrm{E} \otimes p_{S}^{*} \mathcal{N}
$$

for a line bundle $\mathcal{N}$ on $S$. Let $\Gamma: S \hookrightarrow \widehat{X}_{S}$ be the graph of the morphism $\iota \circ \phi: S \rightarrow \widehat{X}$, where $\iota$ is the natural involution of $\widehat{X}$ induced by the operation of taking the dual.

Lemma 2.11 of 4 ] takes now the form: 
Lemma 2.3. In the above situation $\mathbf{S}_{S}^{0}(E)=0$ and $\mathbf{S}_{S}^{1}(E) \otimes \hat{p}_{S}^{*} \mathcal{N} \simeq \Gamma_{*}\left(f^{*} \omega\right)$. In particular, (1) $\mathbf{S}_{\widehat{X}}^{0}(\mathcal{P})=0$ and $\mathbf{S}_{\widehat{X}}^{1}(\mathcal{P}) \simeq \zeta_{*} \hat{p}^{*} \omega$, where $\zeta: \widehat{X} \hookrightarrow \widehat{X} \times_{B} \widehat{X}$ is the graph of the morphism $\iota$.

(2) $\mathbf{S}_{\widehat{X}}^{0}\left(\mathcal{P}^{*}\right)=0$ and $\mathbf{S}_{\widehat{X}}^{1}\left(\mathcal{P}^{*}\right) \simeq \delta_{*} \hat{p}^{*} \omega$, where $\delta: \widehat{X} \hookrightarrow \widehat{X} \times_{B} \widehat{X}$ is the diagonal immersion.

We also recover Corollary 2.12 of [4]:

Corollary 2.4. Let $E$ be a rank-one, zero-degree, torsion-free coherent sheaf on a fibre $X_{t}$. Then

$$
\mathbf{S}_{t}^{0}(E)=0, \quad \mathbf{S}_{t}^{1}(E)=\kappa\left(\left[E^{*}\right]\right)
$$

where $\left[E^{*}\right]$ is the point of $\widehat{X}_{t}$ defined by $E^{*}$.

By reversing the roles of $X$ and $\widehat{X}$, and using the sheaf $\mathcal{Q}=\mathcal{P}^{*} \otimes \pi^{*} p^{*} \omega^{-1}$ instead of $\mathcal{P}$, we define a functor ${ }^{1}$

$$
\begin{aligned}
\widehat{\mathbf{S}}_{S}: D^{-}\left(\widehat{X}_{S}\right) & \rightarrow D^{-}\left(X_{S}\right) \\
G & \mapsto R \pi_{S *}\left(\hat{\pi}_{S}^{*} G \stackrel{L}{\otimes} \mathcal{Q}_{S}\right)
\end{aligned}
$$

and the corresponding functors $\widehat{\mathbf{S}}_{S}^{i}, i=0,1$. Proceeding as in Theorem 3.2 of [4], and taking into account Lemma 2.3, we obtain an invertibility result:

Proposition 2.5. For every $G \in D^{-}\left(\widehat{X}_{S}\right), F \in D^{-}\left(X_{S}\right)$ there are functorial isomorphisms

$$
\mathbf{S}_{S}\left(\widehat{\mathbf{S}}_{S}(G)\right) \simeq G[-1], \quad \widehat{\mathbf{S}}_{S}\left(\mathbf{S}_{S}(F)\right) \simeq F[-1]
$$

in the derived categories $D^{-}\left(\widehat{X}_{S}\right)$ and $D^{-}\left(X_{S}\right)$, respectively.

Proposition 2.6. Let $\mathcal{F}$ be a rank $n$ coherent sheaf on $X_{S}$, flat over $S$, of relative degree d. The relative Chern character of the Fourier-Mukai transform $\mathbf{S}_{S}(\mathcal{F})$ is $(d,-n)$.

Proof. To compute the relative invariants of $\mathbf{S}_{S}(\mathcal{F})$ we take for $S$ a point $t \in B$. The morphism $X_{t} \times \widehat{X}_{t} \rightarrow \widehat{X}_{t}$ is a local complete intersection morphism in the sense of 9 , so that one can apply the Riemann-Roch theorem for singular varieties applies (Corollary 18.3.1 of [9]).

We shall denote by $d(\mathcal{F})$ the relative degree of a sheaf $\mathcal{F}$ and by $\mu(\mathcal{F})=d(\mathcal{F}) / \operatorname{rk}(\mathcal{F})$ its relative slope.

Corollary 2.7. If $\mathcal{F}$ is $W I T_{i}$ and $d \neq 0$, then $\mu(\widehat{\mathcal{F}})=-1 / \mu(\mathcal{F})$.

Corollary 2.8. (1) If $\mathcal{F}$ is WIT , then $d(\mathcal{F}) \geq 0$, and $d(\mathcal{F})=0$ if and only if $\mathcal{F}=0$. (2) If $\mathcal{F}$ is $W I T_{1}$, then $d(\mathcal{F}) \leq 0$.

\footnotetext{
${ }^{1}$ The present definition of the functor $\hat{\mathbf{S}}$ is slightly different than in [4 due to the presence of the factor $p^{*} \omega^{-1}$.
} 
Proceeding as in (11, Theorem 5, or [16]) (cf. also [8], Lemma 3.1), one proves the following Proposition, which allows one to define the rank $n$ Atiyah sheaf on a fibre $X_{t}$.

Proposition 2.9. For every $n \geq 0$, there exists a unique torsion-free indecomposable semistable rank $n$ and degree 0 sheaf $\mathcal{A}_{n}^{(t)}$ on $X_{t}$ which satisfies $H^{0}\left(X_{t}, \mathcal{A}_{n}^{(t)}\right) \neq 0$. Moreover there is an exact sequence

$$
0 \rightarrow \varnothing_{X_{t}} \rightarrow \mathcal{A}_{n}^{(t)} \rightarrow \mathcal{A}_{n-1}^{(t)} \rightarrow 0
$$

Corollary 2.10. The Atiyah sheaf $\mathcal{A}_{n}^{(t)}$ is $W I T_{1}$ and $\mathbf{S}_{t}^{1}\left(\mathcal{A}_{n}^{(t)}\right)=\emptyset_{n \hat{e}(t)}$.

Proof. One applies Corollary 2.4 to the exact sequence in Proposition 2.9 and uses induction.

Then, by the invertibility of the Fourier-Mukai transform, we have $\mathcal{A}_{n}^{(t)}=\hat{\mathbf{S}}_{t}^{0}\left(\varnothing_{n \hat{e}(t)}\right)$. This motivates the following:

Definition 2.11. The relative rank $n$ Atiyah sheaf associated with the fibration $p: X \rightarrow B$ is the sheaf

$$
\mathcal{A}_{n}=\hat{\mathbf{S}}_{B}^{0}\left(\varnothing_{n \Theta}\right) \otimes p^{*} \omega
$$

The factor $p^{*} \omega$ is introduced so that $\mathcal{A}_{1} \simeq \varnothing_{X}$.

\section{Preservation of Stability}

In this section we prove that the Fourier-Mukai transform preserves the (semi)stability of sheaves of positive relative degree. The zero degree case is somehow more delicate and has been treated separately in [10].

When considering sheaves on the total spaces of elliptic fibrations we shall need the notions of "relative torsion-freeness" and "relative (semi)stability". The first means that the sheaf is flat over the base and torsion-free when restricted to any fibre, while the second means that it is pure-dimensional and the restriction to any fibre is $\mu$-(semi)stable in the sense of Simpson [15].

To prove that relatively semistable sheaves of positive degree are $\mathrm{WIT}_{0}$ we need a preliminary result (cf. also [5]).

Lemma 3.1. A coherent sheaf $\mathcal{F}$ on $X$ is WIT $T_{0}$ if and only if

$$
\operatorname{Hom}_{X}\left(\mathcal{F}, \mathcal{P}_{\xi}\right)=0
$$

for every $\xi \in \widehat{X}$.

Proof. By Corollary (2.4), $\mathcal{P}_{\xi}$ is $\mathrm{WIT}_{1}$ and $\mathbf{S}^{1}\left(\mathcal{P}_{\xi}\right)=\kappa\left(\xi^{*}\right)$, where $\xi^{*}$ is the point of $\widehat{X}_{t}$ corresponding to $\mathcal{P}_{\xi}^{*}(t=\hat{p}(\xi))$. Then, Parseval theorem [4, Prop. 3.4] implies that

$$
\operatorname{Hom}_{X}\left(\mathcal{F}, \mathcal{P}_{\xi}\right) \simeq \operatorname{Hom}_{D(\widehat{X})}\left(\mathbf{S}(\mathcal{F}), \kappa\left(\xi^{*}\right)[1]\right) .
$$


If $\mathcal{F}$ is not $\mathrm{WIT}_{0}$, there is a point $\xi^{*} \in \widehat{X}$ such that a nonzero morphism $\mathbf{S}^{1}(\mathcal{F}) \rightarrow \kappa\left(\xi^{*}\right)$ exists. This gives rise to a non-zero morphism $\mathbf{S}(\mathcal{F}) \rightarrow \kappa\left(\xi^{*}\right)[1]$ in the derived category, so that $\operatorname{Hom}_{X}\left(\mathcal{F}, \mathcal{P}_{\xi}\right) \neq 0$. The converse is straightforward.

Theorem 3.2. Let $\mathcal{F}$ be a relatively (semi)stable sheaf on $X$, flat over $B$, with $d(\mathcal{F})>0$. Then $\mathcal{F}$ is WIT $T_{0}$ and its Fourier-Mukai transform $\widehat{\mathcal{F}}$ is relatively (semi)stable.

Proof. We deal with the semistable case, the stable case differing only by some inequalities which become strict. By base change it is enough to restrict to a fibre $X_{t}$. The fact that $\mathcal{F}$ is $\mathrm{WIT}_{0}$ follows from Lemma 3.1 since $\mathcal{F}$ is semistable of positive degree.

The sheaf $\widehat{\mathcal{F}}=\mathbf{S}_{t}^{0}(\mathcal{F})$ has rank $d(\mathcal{F})>0$ by Proposition 2.6 and is $\mathrm{WIT}_{1}$. All of its subsheaves are $\mathrm{WIT}_{1}$ as well so they cannot have zero-dimensional support; it follows that $\widehat{\mathcal{F}}$ is torsion-free. Let us consider an exact sequence

$$
0 \rightarrow \mathcal{E} \rightarrow \widehat{\mathcal{F}} \rightarrow \mathcal{G} \rightarrow 0
$$

with $\mathcal{G}$ semistable. By applying the inverse Fourier-Mukai transform one obtains that $\mathcal{E}$ is $\mathrm{WIT}_{1}$ and that there is an exact sequence

$$
0 \rightarrow \widehat{\mathbf{S}}_{t}^{0} \mathcal{G} \rightarrow \widehat{\mathcal{E}} \rightarrow \mathcal{F} \rightarrow \widehat{\mathbf{S}}_{t}^{1} \mathcal{G} \rightarrow 0
$$

which splits into

$$
0 \rightarrow \widehat{\mathbf{S}}_{t}^{0} \mathcal{G} \rightarrow \widehat{\mathcal{E}} \rightarrow \mathcal{N} \rightarrow 0, \quad 0 \rightarrow \mathcal{N} \rightarrow \mathcal{F} \rightarrow \widehat{\mathbf{S}}_{t}^{1} \mathcal{G} \rightarrow 0 .
$$

Then $\widehat{\mathbf{S}}_{t}^{1} \mathcal{G}$ and $\mathcal{N}$ are $\mathrm{WIT}_{0}$, and we obtain exact sequences

$$
0 \rightarrow \mathcal{E} \rightarrow \widehat{\mathcal{N}} \rightarrow \mathbf{S}_{t}^{1} \widehat{\mathbf{S}}_{t}^{0} \mathcal{G} \rightarrow 0, \quad 0 \rightarrow \widehat{\mathcal{N}} \rightarrow \widehat{\mathcal{F}} \rightarrow \mathbf{S}_{t}^{0} \widehat{\mathbf{S}}_{t}^{1} \mathcal{G} \rightarrow 0
$$

so that $\widehat{\mathbf{S}}_{t}^{0} \mathcal{G}$ is $\mathrm{WIT}_{1}$, and one has

$$
0 \rightarrow \mathbf{S}_{t}^{1} \widehat{\mathbf{S}}_{t}^{0} \mathcal{G} \rightarrow \mathcal{G} \rightarrow \mathbf{S}_{t}^{0} \widehat{\mathbf{S}}_{t}^{1} \mathcal{G} \rightarrow 0
$$

If $\mathbf{S}_{t}^{1} \widehat{\mathbf{S}}_{t}^{0} \mathcal{G} \neq 0$ the semistability of $\mathcal{G}$ implies that $d\left(\mathbf{S}_{t}^{1} \widehat{\mathbf{S}}_{t}^{0} \mathcal{G}\right)<0$ which contradicts Corollary 2.8 since $\mathbf{S}_{t}^{1} \widehat{\mathbf{S}}_{t}^{0} \mathcal{G}$ is WIT $_{0}$. Then $\mathbf{S}_{t}^{1} \widehat{\mathbf{S}}_{t}^{0} \mathcal{G}=0$ and we have $\mathcal{E} \simeq \widehat{\mathcal{N}}$, so that $\widehat{\mathcal{E}} \simeq \mathcal{N}$ and $\mu(\widehat{\mathcal{E}}) \leq \mu(\mathcal{F})$ since $\mathcal{F}$ is semistable. By Corollary 2.8, $d(\mathcal{E}) \leq 0$.

We can exclude the case $d(\mathcal{E})=0$ (otherwise $\widehat{\mathcal{E}}$ is a subsheaf of $\mathcal{F}$ of rank zero). Finally, if $d(\mathcal{E})<0$ we can apply Corollary 2.7 to obtain $\mu(\mathcal{E}) \leq \mu(\widehat{\mathcal{F}})$ which proves the semistability of $\widehat{\mathcal{F}}$.

Corollary 3.3. Let $\mathcal{F}$ be a torsion-free semistable sheaf on $X_{t}$ of degree $d>0$. Then $H^{1}\left(X_{t}, \mathcal{F} \otimes \mathcal{P}_{\xi}\right)=0$ for every $\xi \in \widehat{X}_{t}$.

As a side result we show that the relative Fourier-Mukai transform provides a characterization of some moduli spaces of relatively stable bundles. Let $J_{n} \rightarrow B$ be the relative Jacobian of invertible sheaves of relative degree $n$ and $\bar{J}_{n} \rightarrow B$ the natural compactification of $J_{n}$ obtained by adding to $J_{n}$ the $B$-flat coherent sheaves on $p: X \rightarrow B$ whose restrictions to the fibres of are torsion-free, of rank one and degree $n$ [2]. Theorem 3.2 gives: 
Corollary 3.4. Let $\mathcal{N}$ be an invertible sheaf on $X$ of relative degree $m$, and let $\mathcal{M}(n$, $n m-1)$ be the moduli space of rank $n$ relatively $\mu$-stable sheaves on $X \rightarrow B$ of degree $n m-1$. The Fourier-Mukai transform induces an isomorphism of $B$-schemes

$$
\begin{aligned}
& \bar{J}_{n} \stackrel{\mathbf{S}^{0} \otimes \mathcal{N}}{\longrightarrow} \mathcal{M}(n, n m-1) \\
& E \quad \mapsto \quad \mathbf{S}^{0}(E) \otimes \mathcal{N} .
\end{aligned}
$$

\section{Flag schemes and Hecke correspondences}

As a first application of the above results we construct the relative Hecke correspondences over an elliptic fibration, showing also how, via Fourier-Mukai transform, they relate to some varieties parametrizing flags of relatively stable sheaves.

4.1. Flag schemes. We consider exact sequences

$$
0 \rightarrow \mathrm{L} \otimes \mathcal{A}_{i}^{(t)} \rightarrow \mathcal{E}_{n} \rightarrow \mathcal{E}_{n-i} \rightarrow 0
$$

of sheaves on a fibre $X_{t}$, where $\mathrm{E}$ is torsion-free, of rank 1 and degree zero, $\mathcal{A}_{i}^{(t)}$ is the rank $i$ Atiyah sheaf on $X_{t}$ and $\mathcal{E}_{n}, \mathcal{E}_{n-i}$ are torsion-free and stable of degree $d \geq 0$ and rank $n$ and $n-i$, respectively.

We construct a moduli scheme $F^{i}(n, d)$ parametrizing such exact sequences. The relevant functor of points is defined as follows: for every $B$-scheme $S \rightarrow B$, let $\mathbf{F}^{i}(n, d)(S)$ be the set of all exact sequences

$$
0 \rightarrow \mathrm{E} \otimes \mathcal{A}_{i} \rightarrow \mathcal{E}_{n} \rightarrow \mathcal{E}_{n-i} \rightarrow 0
$$

of sheaves on $X_{S} \rightarrow S$, where $\mathrm{E}$ is relatively torsion-free rank 1 and zero degree, $\mathcal{A}_{i}$ is the rank $i$ relative Atiyah sheaf (Definition 2.11), and $\mathcal{E}_{n}, \mathcal{E}_{n-i}$ are relatively torsion-free and stable sheaves of degree $d \geq 0$ and $\operatorname{rank} n, n-i$ respectively. Two such sequences

$$
\begin{aligned}
0 & \rightarrow \mathrm{L} \otimes \mathcal{A}_{i} \rightarrow \mathcal{E}_{n} \rightarrow \mathcal{E}_{n-i} \rightarrow 0 \\
0 & \rightarrow \mathrm{E}^{\prime} \otimes \mathcal{A}_{i} \rightarrow \mathcal{E}_{n}^{\prime} \rightarrow \mathcal{E}_{n-i}^{\prime} \rightarrow 0
\end{aligned}
$$

are considered to be equivalent if there exist line bundles $\mathrm{E}_{1}, \mathrm{E}_{2}$ on $S$ such that the first sequence is isomorphic to

$$
0 \rightarrow \mathrm{E}^{\prime} \otimes \mathcal{A}_{i} \otimes p_{S}^{*} \mathrm{~L}_{1} \rightarrow \mathcal{E}_{n}^{\prime} \otimes p_{S}^{*} \mathrm{\complement}_{2} \rightarrow \mathcal{E}_{n-i}^{\prime} \rightarrow 0
$$

in the obvious sense.

We assume that $n, d$ are coprime, so that $\mathcal{M}(n, d)$ is a fine moduli scheme (consisting only of stable sheaves) and there is a universal sheaf $U_{n, d}$ on $X \times_{B} \mathcal{M}(n, d) \rightarrow \mathcal{M}(n, d)$.

Proposition 4.1. If $n$ and $d$ are coprime, there is a B-scheme $F^{i}(n, d) \rightarrow B$ which represents the functor $\mathbf{F}^{i}(n, d)$. 
Proof. Let us write $Y=\widehat{X} \times_{B} \mathcal{M}(n, d)$, so that $Y_{X}=X \times_{B} \widehat{X} \times_{B} \mathcal{M}(n, d)$, and denote by $\pi_{j k}$ the projection of $Y_{X}$ onto the product of the $j$ th and $k$ th factors. By (1.1) of [2] there exists a coherent sheaf $\mathcal{H}$ on $Y$ such that for every morphism $S \rightarrow Y$ and every quasi-coherent sheaf $\mathcal{F}$ on $S$ one has

$$
\operatorname{Hom}_{S}\left(\mathcal{H}_{S}, \mathcal{F}\right) \simeq \operatorname{Hom}_{X_{S}}\left(\left(\pi_{12}^{*} \mathcal{P} \otimes \pi_{1}^{*} \mathcal{A}_{i}\right)_{S}, \pi_{13}^{*} U_{n, d} \otimes_{S} \mathcal{F}\right)
$$

functorially in $\mathcal{F}$.

If $q: P=\operatorname{Proj} S(\mathcal{H}) \rightarrow Y$ is the associated Grothendieck projective bundle, there is a universal epimorphism $q^{*} \mathcal{H} \rightarrow \varnothing_{P}(1)$. According to Eq. (5) this yields a universal morphism of sheaves on $X_{P}=X \times_{B} P \rightarrow P$

$$
\left(\pi_{12}^{*} \mathcal{P} \otimes \pi_{1}^{*} \mathcal{A}_{i}\right)_{P} \stackrel{\phi}{\longrightarrow} \pi_{13}^{*} U_{n, d} \otimes_{P} \emptyset_{P}(1) .
$$

By (2.3) of 2] there is a subscheme $V$ of $P$ parametrizing the points $T \rightarrow P$ such that coker $\phi_{T}$ is flat over $T$ and relatively torsion-free of rank $n-r$. The subscheme of $V$ determined by the points where coker $\phi$ is semistable is the desired scheme $F^{r}(n, d)$.

We call $F^{i}(n, d)$ the flag scheme of order $i$ of $p: X \rightarrow B$. We have scheme morphisms

$$
\widehat{X} \times_{B} \mathcal{M}(n, d) \stackrel{\pi_{n}}{\longleftarrow} F^{i}(n, d) \stackrel{\varphi_{n}}{\longrightarrow} \mathcal{M}(n-i, d)
$$

defined by $\pi_{n}\left(0 \rightarrow \mathrm{E} \otimes \mathcal{A}_{i} \rightarrow \mathcal{E}_{n} \rightarrow \mathcal{E}_{n-i} \rightarrow 0\right)=\left(\mathrm{E}, \mathcal{E}_{n}\right), \varphi_{n}\left(0 \rightarrow \mathrm{E} \otimes \mathcal{A}_{i} \rightarrow \mathcal{E}_{n} \rightarrow \mathcal{E}_{n-i} \rightarrow\right.$ $0)=\mathcal{E}_{n-i}$.

4.2. Relative Hecke correspondences. Let $\mathcal{G}_{r}$ be a torsion-free sheaf on $X$ of rank $r$ and relative degree $m<0$.

Definition 4.2. A higher modification of length $i \leq r$ at a point $x \in X$ is an exact sequence

$$
0 \rightarrow \mathcal{G}_{r} \rightarrow \mathcal{G}_{r}^{\prime} \rightarrow \mathcal{K}(x) \rightarrow 0
$$

where $\mathcal{G}_{r}^{\prime}$ is a torsion-free sheaf of rank $r$ and $\mathcal{K}(x)$ is a coherent torsion sheaf of length $i$ concentrated at $x$.

The sheaf $\mathcal{G}_{r}^{\prime}$ has degree $m-i<0$. We shall construct a $B$-scheme $H^{i}(r, m)$ parametrizing the length $i$ higher modifications.

We define now the relevant functor of points. For every morphism $S \rightarrow B$ we let $\mathbf{H}^{i}(r, m)(S)$ be the family of all exact sequences

$$
0 \rightarrow \mathcal{G}_{r} \rightarrow \mathcal{G}_{r}^{\prime} \rightarrow \mathcal{K}_{i}(x) \rightarrow 0
$$

of $S$-flat sheaves on $X_{S}$; here $\mathcal{G}_{r}, \mathcal{G}_{r}^{\prime}$ are relatively stable sheaves of rank $r$ and negative degree $m, m-i$ respectively, while $\mathcal{K}_{i}(x)$ has length $i$ on every fibre and is concentrated on the image $x(S)$ of a section $x: S \hookrightarrow X_{S}$ of $p_{S}$. Two such sequences

$$
\begin{aligned}
0 & \rightarrow \mathcal{G}_{r} \rightarrow \mathcal{G}_{r}^{\prime} \rightarrow \mathcal{K}_{i}(x) \rightarrow 0 \\
0 & \rightarrow \overline{\mathcal{G}}_{r} \rightarrow \overline{\mathcal{G}}_{r}^{\prime} \rightarrow \overline{\mathcal{K}}_{i}(x) \rightarrow 0,
\end{aligned}
$$


are considered equivalent if there exist line bundles $\mathrm{E}_{1}, \mathrm{E}_{2}$ over $S$ such that

$$
0 \rightarrow \mathcal{G}_{r} \rightarrow \mathcal{G}_{r}^{\prime} \rightarrow \mathcal{K}_{i}(x) \rightarrow 0
$$

is isomorphic to

$$
0 \rightarrow \overline{\mathcal{G}}_{r} \otimes p_{S}^{*} \mathrm{E}_{2} \rightarrow \overline{\mathcal{G}}_{r}^{\prime} \rightarrow \overline{\mathcal{K}}_{i}(x) \otimes p_{S}^{*} \mathrm{E}_{1} \rightarrow 0 .
$$

Let us consider integers $d>0, n \geq i \geq 0$.

Theorem 4.3. The Fourier-Mukai transform induces an isomorphism of functors

$$
\mathbf{S}: \mathbf{F}^{i}(n, d) \simeq \mathbf{H}^{i}(d,-n) \text {. }
$$

Proof. The Fourier-Mukai transform interchanges the exact sequences (41) and (17).

Corollary 4.4. If $n, d$ are coprime, the functor $\mathbf{H}^{i}(d,-n)$ is representable by a B-scheme $H^{i}(d,-n)$, called the length $i$ Hecke correspondence. The Fourier-Mukai transforms yields a scheme isomorphism

$$
\mathbf{S}: F^{i}(n, d) \simeq H^{i}(d,-n)
$$

between the flag scheme and the length $i$ Hecke correspondence.

The reason for giving $H^{i}(d,-n)$ this name is that for every point $x \in X$, the fibre $H_{x}^{i}(d,-n)$ is the graph of the classical length $i$ Hecke correspondence between the moduli spaces $\mathcal{M}\left(X_{t}, d,-n\right)$ and $\mathcal{M}\left(X_{t}, d, i-n\right)$ on the curve $X_{t}(t=p(x))$ (cf. [7, 11]).

There are scheme morphisms

$$
X \times_{B} \mathcal{M}(d,-n) \stackrel{\alpha_{i}}{\longleftarrow} H^{i}(d,-n) \stackrel{\beta_{i}}{\longrightarrow} \mathcal{M}(d, i-n)
$$

defined by

$$
\begin{gathered}
\alpha_{i}\left(0 \rightarrow \mathcal{G}_{d} \rightarrow \mathcal{G}_{d}^{\prime} \rightarrow \mathcal{K}_{i}(x) \rightarrow 0\right)=\left(x, \mathcal{G}_{d}\right), \\
\beta_{i}\left(0 \rightarrow \mathcal{G}_{d} \rightarrow \mathcal{G}_{d}^{\prime} \rightarrow \mathcal{K}_{i}(x) \rightarrow 0\right)=\mathcal{G}_{d}^{\prime} .
\end{gathered}
$$

The Fourier-Mukai transform maps isomorphically diagram (6) to diagram (8)):

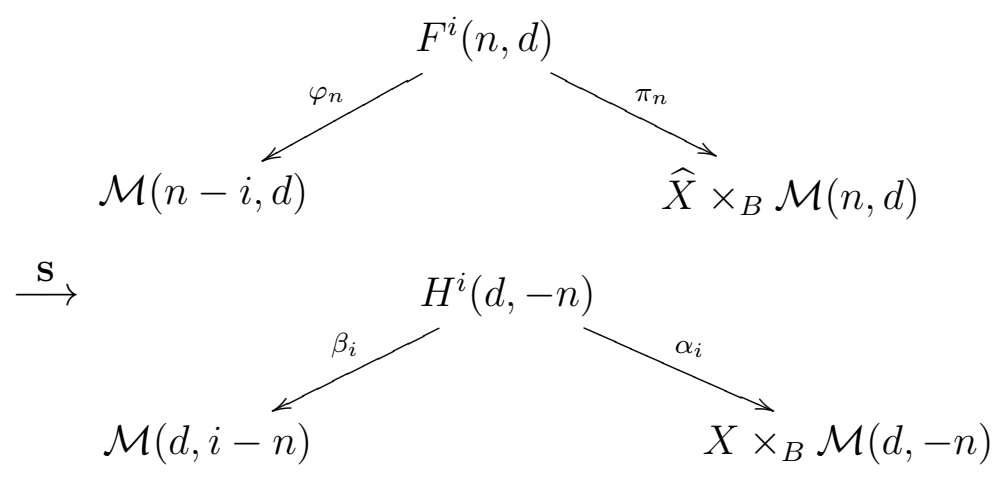

The isomorphism between $\widehat{X} \times{ }_{B} \mathcal{M}(n, d)$ and $X \times_{B} \mathcal{M}(d,-n)$ is provided by the morphism $\left(\varpi^{-1} \circ \iota, \mathbf{S}^{0}\right)$, where $\iota: \widehat{X} \rightarrow \widehat{X}$ is the morphism that maps $\xi=[\mathrm{E}]$ into $\xi^{*}=\left[\mathrm{E}^{*}\right]$, while the isomorphism between $\mathcal{M}(n-i, d)$ and $\mathcal{M}(d, i-n)$ is induced by $\mathbf{S}^{0}$. 
Remark 4.5. This result amounts to a generalization to the elliptic relative case of the geometric Langlands correspondence constructed by Laumon 11] in the case of curves over a field. In Drinfeld's terminology, Theorem 4.3 means that the Fourier-Mukai transform induces a duality between the operators $S$ and $T_{p}$ as defined in [7].

\section{REFERENCES}

[1] Atiyah, M.F., Vector bundles over an elliptic curve, Proc. London Math. Soc. 7 (1957), 414-452.

[2] Altman, A., and Kleiman, S., Compactifying the Picard scheme, Adv. Math. 35 (1980), 50-112.

[3] Bartocci, C., Bruzzo, U., and Hernández Ruipérez, D., A Fourier-Mukai transform for stable bundles on K3 surfaces, J. reine angew. Math. 486 (1997), 1-16.

[4] Bartocci, C., Bruzzo, U., Hernández Ruipérez, D., and Muñoz Porras, J.M., Mirror symmetry on K3 surfaces via Fourier-Mukai transform, Commun. Math. Phys. 195 (1998), 79-93.

[5] Bridgeland, T., Fourier-Mukai transforms for elliptic surfaces, J. reine angew. Math. 498 (1998), $115-133$.

[6] Bridgeland, T., and Maciocia, A., Fourier-Mukai transforms for K3 fibrations, math.AG/9908022.

[7] Drinfeld, V.G., Two-dimensional $\ell$-adic representations of the fundamental group of a curve over a finite field and automorphic forms on GL(2), Amer. J. Math. 105 (1983), 85-114.

[8] Friedman, R., Morgan, J. W., and Witten, E., Vector bundles over elliptic fibrations, J. Alg. Geom. 8 (1999), 279-401.

[9] Fulton, W., "Intersection Theory," Springer-Verlag, Berlin (1984).

[10] Hernández Ruipérez, D., and Muñoz Porras, J.M., Structure of the moduli space of stable sheaves on elliptic surfaces, Preprint Universidad de Salamanca.

[11] Laumon, G., Correspondance de Langlands géométrique pour les corps de fonctions, Duke Math. J. 54 (1987), 309-359.

[12] Mukai, S. Duality between $D(X)$ and $D(\hat{X})$ with its application to Picard sheaves, Nagoya Math. J. 81 (1981), 153-175.

[13] _ Fourier functor and its application to the moduli of bundles on an abelian variety, Advanced Studies in Pure Mathematics, Algebraic Geometry Conference, Sendai 1985, 10 (1987), 515-550.

[14] Duality of polarized K3 surfaces, in "New trends in algebraic geometry" (Warwick, 1996), p. 311-326, London Math. Soc. Lecture Note Ser. 264, Cambridge Univ. Press, Cambridge 1999.

[15] Simpson, C., Moduli of representations of the fundamental group of a smooth projective variety, Publ. Math. I.H.E.S. 79 (1994), 47-129.

[16] Tu, L. W, Semistable bundles over an elliptic curve, Adv. Math. 98 (1993), 1-26. 